\title{
Cation order control of correlations in double perovskite $\mathrm{Sr}_{2} \mathrm{VNbO}_{6}$
}

\author{
Arpita Paul๑ and Turan Birolø* \\ Department of Chemical Engineering and Materials Science, University of Minnesota, Minneapolis, Minnesota 55455, USA
}

(Received 9 December 2019; revised 21 May 2020; accepted 25 June 2020; published 28 July 2020)

\begin{abstract}
Double perovskites extend the design space for new materials and they often host phenomena that do not exist in their parent perovskite compounds. Here we present a detailed first-principles study of the correlated double perovskite $\mathrm{Sr}_{2} \mathrm{VNbO}_{6}$, where the intercationic charge transfer and strength of electronic correlations depend strongly on the cation order. By using density functional theory + embedded dynamical mean field theory, we show that this compound has a completely different electronic structure than either of its parent compounds despite $\mathrm{V}$ and $\mathrm{Nb}$ being from the same group in the Periodic Table. We explain how the electronic correlations' effect on the crystal structural parameters determines on which side of the Hund's metal-Mott insulator transition the material is. Our results demonstrate the emergence of Hund's metallic behavior in a double perovskite that has $d^{1}$ parents and underline the importance of electronic correlation effects on the crystal structure.
\end{abstract}

DOI: 10.1103/PhysRevResearch.2.033156

\section{INTRODUCTION}

Transition metal oxides (TMOs) are the focus of great interest as they host a wide variety of electronic phenomena including metal-insulator transitions, charge orbital, or spin ordering, multiferroicity, colossal magnetoresistance, different types of magnetism, and high-temperature superconductivity [1-4]. Many of these emergent properties and rich phase diagrams arise from the interplay between charge, spin, orbital, and lattice degrees of freedom. Strong Coulomb interactions between electrons in the $d$ orbitals of transition metals often lead to strong electronic correlations, and the directional nature of these orbitals leads to strong coupling between the electronic wave function and the lattice degrees of freedom [5]. This makes it possible for seemingly very similar compounds to have very different properties that can be tuned easily via external fields or chemical substitution.

Perhaps the best demonstration of the richness of TMOs is provided by the perovskite structure: Almost any phenomenon observed in the solid state is realized in at least one $\mathrm{ABO}_{3}$ perovskite oxide. The phase diagrams of perovskites can be further expanded by considering double perovskites, where one of the cations is partially substituted in an ordered fashion [6,7]. For example, in the most common form of $B$-site ordered $A_{2} B B^{\prime} \mathrm{O}_{6}$ double perovskites, there are two transition metals on the $B$ site that are alternating at every other unit cell [Fig. 1(a)]. Multiple $B$-site transition metal cations provide one more degree of freedom to realize different electronic phases such as the rare combination of ferromagnetism with insulating behavior that is rather commonplace in double

\footnotetext{
*tbirol@umn.edu

Published by the American Physical Society under the terms of the Creative Commons Attribution 4.0 International license. Further distribution of this work must maintain attribution to the author(s) and the published article's title, journal citation, and DOI.
}

perovskites like $\mathrm{La}_{2} \mathrm{MnNiO}_{6}$ [8]. Other phenomena such as multiferroicity, frustrated antiferromagnetism, magneto-optic properties, and spin liquid related phenomena have been observed in double perovskites with parent compounds that do not display the same properties [9-13].

The ordering of different metal ions in double perovskites greatly affects electronic and structural properties [7]. For example, the degree of cation order often determines the strength of relaxor characteristics that ferroelectric double perovskites display [14] and the ferromagnetic properties of the half-metal $\mathrm{Sr}_{2} \mathrm{FeMoO}_{6}$ are affected significantly by cation disorder $[15,16]$. It is also possible to have different types of cation orders, where the $B$ and $B^{\prime}$ ions arrange themselves in a rocksalt, layered, or columnar fashion [7], and a particular stoichiometry can in principle give rise to very different properties depending on the cation order because of the different connectivity of $\mathrm{BO}_{6}$ octahedra. (The rocksalt ordering [Fig. 1(a)] gives rise to $\mathrm{BO}_{6}$ octahedra that are isolated from each other, whereas the layered ordering [Fig. 1(b)] gives rise to extended planes of connected $\mathrm{BO}_{6}$ octahedra.)

In this first-principles study, we focus on the seemingly simple double perovskite $\mathrm{Sr}_{2} \mathrm{VNbO}_{6}$. Both parent compounds of $\mathrm{Sr}_{2} \mathrm{VNbO}_{6}\left(\mathrm{SrVO}_{3}\right.$ and $\left.\mathrm{SrNbO}_{3}\right)$ are metallic perovskites with a single electron in partially filled $d$ shells of their $B$-site cations $\left(\mathrm{V}^{4+}\right.$ and $\left.\mathrm{Nb}^{4+}\right)$ [17]. Using density functional theory + embedded dynamical mean field theory (DFT+DMFT) [18-20], we show that this double perovskite has an electronic structure that is strongly intertwined with the cation order and crystal structural parameters (bond-length disproportionation) in an exceedingly sensitive fashion. Even though $\mathrm{V}$ and $\mathrm{Nb}$ come from the same group in the Periodic Table, the difference between their electronegativities leads to an almost complete transfer of an electron from $\mathrm{Nb}$ to $\mathrm{V}$ in $\mathrm{Sr}_{2} \mathrm{VNbO}_{6}$, which results in formal valences closer to $\mathrm{Nb}^{5+}$ and $\mathrm{V}^{3+}$ instead of $\mathrm{Nb}^{4+}$ and $\mathrm{V}^{4+}$. The degree of this intercationic electron transfer depends on the type of the cation order (layered vs rocksalt) present in the system. Depending on this cation order and the resulting changes in the bandwidths, 
(a) $r-\mathrm{Sr}_{2} \mathrm{VNbO}_{6}$

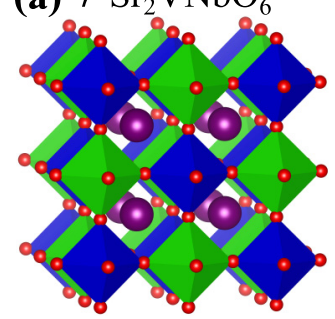

(b) $l-\mathrm{Sr}_{2} \mathrm{VNbO}_{6}$
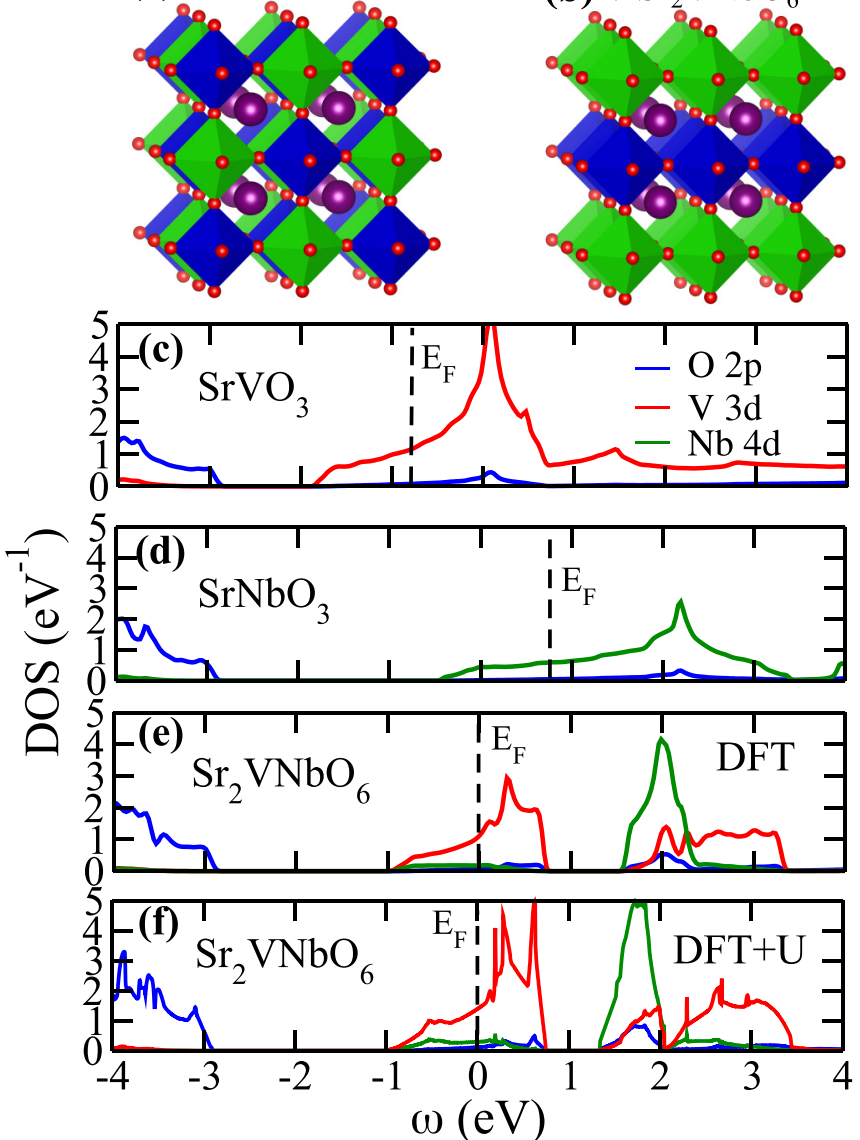

FIG. 1. (a) Rocksalt and (b) layered ordered double perovskite $\mathrm{Sr}_{2} \mathrm{VNbO}_{6}$. The $\mathrm{VO}_{6}$ and $\mathrm{NbO}_{6}$ octahedra are presented in blue (dark) and green (light). (c) Density of states of $\mathrm{SrVO}_{3}$, (d) $\mathrm{SrNbO}_{3}$, and (e) rocksalt ordered $\mathrm{Sr}_{2} \mathrm{VNbO}_{6}$ calculated using DFT. The O $2 p$ levels in different compounds are aligned with each other. (f) Nonspin-polarized DFT $+U$ calculations on the rocksalt ordered double perovskite give qualitatively similar densities of states to calculations with no $U$.

the double perovskite $\mathrm{Sr}_{2} \mathrm{VNbO}_{6}$ behaves as either a strongly correlated metal, which has part of its electronic correlations induced by the on-site Hund's coupling $J$ [21,22], or a Mott insulator, despite the fact that both parent compounds $\mathrm{SrVO}_{3}$ and $\mathrm{SrNbO}_{3}$ are mildly correlated Fermi liquids. Our results also underline the effect of the paramagnetic moments on the crystal structure and show that in order to get the Mott insulating phase the crystal structure parameters need to be calculated using DFT+DMFT, rather than DFT or DFT $+U$ as is commonly done due to the computational cost of structural predictions from DFT+DMFT.

\section{METHODS}

Determination of lattice constants and internal atomic coordinates at the DFT $+U$ level is performed using using density functional theory and projector augmented wave formalism as implemented in the Vienna ab initio simulation package unless stated otherwise [23,24]. DFT $+U$ correction was employed for both transition metals with
$U=4 \mathrm{eV}$ for $\mathrm{V}$ and $U=1 \mathrm{eV}$ for $\mathrm{Nb}$, along with the PerdewBurke-Ernzerhof generalized gradient approximation revised for solids (PBEsol) [25]. These values of $U$ were obtained by calculating the bulk lattice constants of non-spin-polarized $\mathrm{SrVO}_{3}$ and $\mathrm{SrNbO}_{3}$ that match the experimentally reported values [26-28]. $8 \times 8 \times 8$ and $8 \times 8 \times 4$ uniform meshes of $k$ points were used to compute Brillouin zone integrations for the primitive cells of rocksalt ordered and layered ordered compounds. Our optimized lattice constant of rocksalt ordered $\mathrm{Sr}_{2} \mathrm{VNbO}_{6}$ is $7.8705 \AA$ and lattice constants of layered ordered $\mathrm{Sr}_{2} \mathrm{VNbO}_{6}$ are $a=3.937 \AA$ and $c=7.928 \AA$. Note that the estimated lattice constant of the rocksalt ordered structure is close to the average of the lattice constants of its parent compounds.

The DFT band structures reported are calculated using the linearized augmented plane wave approach as implemented in the WIEN2K code [29] and no DFT $+U$ correction. The DFT+DMFT calculations are performed using the eDMFT software package $[18,30]$ using the continuous time quantum Monte Carlo impurity solver [31,32]. All DMFT calculations are performed at $290 \mathrm{~K}$ and with the exact double counting scheme [33]. Both $\mathrm{V}$ and $\mathrm{Nb}$ atoms are treated as impurities with on-site Hubbard $U$ values of $U=10 \mathrm{eV}$ for $\mathrm{V}$ and $U=6 \mathrm{eV}$ for $\mathrm{Nb}$. These values are significantly higher than the ones used for DFT $+U$ because of the different screening processes taken explicitly into account in DFT+DMFT calculations. We note that the suitable $U$ values are also implementation dependent: Our values are larger than typical values used for different DFT+DMFT implementations that use a Wannier-based approach rather than a local-projectorbased one. These larger values are explicitly and extensively tested for various (especially $3 d$ ) transition metals and are known to capture quantities such as bandwidth renormalization correctly $[17,34,35]$. The on-site Hund's $J$ value, on the other hand, is not strongly screened and is expected to be very similar in all of these methods. We use $J=0.7 \mathrm{eV}$, except in cases where we repeat calculations with different values of $J$ to see how the strength of correlations depends on it. All of the reported DFT, DFT $+U$, and DFT + DMFT calculations preserve time-reversal symmetry and hence correspond to paramagnetic or diamagnetic phases, which are expected at room temperature.

\section{RESULTS}

\section{A. Rocksalt ordered double perovskite}

Both $\mathrm{SrVO}_{3}$ and $\mathrm{SrNbO}_{3}$ have the cubic perovskite structure with no structural distortions such as octahedral rotations at room temperature $[26,27]$. Their $t_{2 g}$ bands are well separated from the oxygen $p$ bands by $\sim 1 \mathrm{eV}$ in $\mathrm{SrVO}_{3}$ and $\sim 2.5 \mathrm{eV}$ in $\mathrm{SrNbO}_{3}$, as shown in Figs. 1(c) and 1(d). This separation is determined by a combination of the bandwidths, crystal field splittings, and the electronegativities of $\mathrm{V}$ and $\mathrm{Nb}$ cations: $\mathrm{V}^{4+}$ is more electronegative than $\mathrm{Nb}^{4+}$ [36], and hence $d$ bands of $\mathrm{V}$ are lower in energy than those of $\mathrm{Nb}$. This large difference between the energies of the $t_{2 g}$ bands in the parent compounds lead to an interesting electronic structure in the rocksalt ordered double perovskite $\mathrm{Sr}_{2} \mathrm{VNbO}_{6}$. In Fig. 1(e) we show the density of states of the rocksalt 


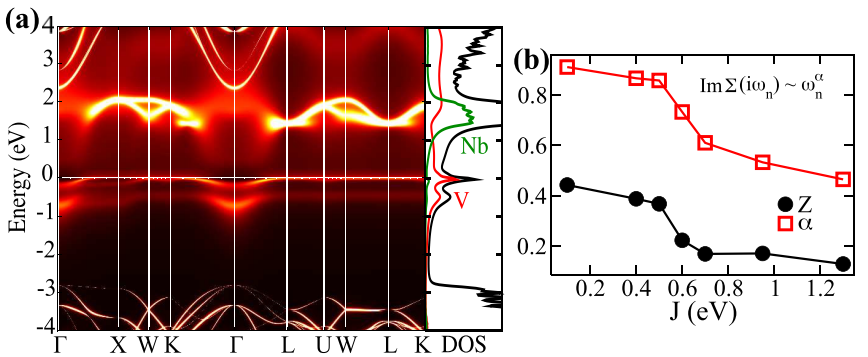

FIG. 2. (a) Spectral function $S(k, \omega)$ and density of states of rocksalt ordered $r$ - $\mathrm{Sr}_{2} \mathrm{VNbO}_{6}$ at room temperature calculated using DFT+DMFT. (b) Quasiparticle weight $Z$ and the exponent of the imaginary part of the self-energy on the imaginary axis $\alpha$ of $r-\mathrm{Sr}_{2} \mathrm{VNbO}_{6}$ at room temperature for varying degrees of on-site Hund's coupling $J$. Hund's coupling in the range of $\sim 0.7-0.9 \mathrm{eV}$ is expected to describe the $\mathrm{V}$ ion well.

ordered $\mathrm{Sr}_{2} \mathrm{VNbO}_{6}$, henceforth referred to as $r-\mathrm{Sr}_{2} \mathrm{VNbO}_{6}$, at the DFT level calculated using the DFT $+U$ optimized crystal structure. Only the vanadium $t_{2 g}$ bands cross the Fermi level and the niobium $d$ shell is formally empty. An electron is transferred from $\mathrm{Nb}$ to $\mathrm{V}$ compared to the parent perovskites; in other words, instead of the $4+$ valences of transition metals in $\mathrm{SrV}^{4+} \mathrm{O}_{3}$ and $\mathrm{SrNb}^{4+} \mathrm{O}_{3}$, the double perovskite has $\mathrm{Sr}_{2} \mathrm{~V}^{3+} \mathrm{Nb}^{5+} \mathrm{O}_{6}$. [Use of DFT $+U$ without breaking timereversal symmetry does not change the main features of the DOS, as shown in Fig. 1(f).]

Transfer of charge between $B$-site cations in $A_{2} B B^{\prime} \mathrm{O}_{6}$ double perovskites is not uncommon [6,37]. For example, Mo is in a higher valence state in $\mathrm{Sr}_{2} \mathrm{FeMoO}_{6}$ and $\mathrm{Sr}_{2} \mathrm{VMoO}_{6}$ than in $\mathrm{SrMoO}_{3}[38,39]$. In $\mathrm{Ba}_{2} \mathrm{VFeO}_{6}$, Mott multiferroicity is predicted to emerge as a result of the charge transfer from $\mathrm{V}$ to $\mathrm{Fe}[40]$. Short-period $\left(\mathrm{LaTiO}_{3}\right)_{1} /\left(\mathrm{LaNiO}_{3}\right)_{1}$ heterostructures, which can be considered as layered double perovskites, also display similar charge transfer [41]. While, unlike these examples, $\mathrm{V}$ and $\mathrm{Nb}$ are from the same group, a significant difference in the charge states of $\mathrm{V}$ and $\mathrm{Nb}$ is experimentally observed in $\mathrm{SrV}_{1-x} \mathrm{Nb}_{x} \mathrm{O}_{3}$ solid solutions as well [42], but any implications thereof in ordered $\mathrm{Sr}_{2} \mathrm{VNbO}_{6}$ double perovskites have not been addressed in the literature yet.

In order to predict the electronic structure of $\mathrm{Sr}_{2} \mathrm{VNbO}_{6}$ at room temperature, we performed DFT + embedded dynamical mean field theory (DFT+eDMFT) calculations $[18,19]$. A simple measure of electronic correlation strength, especially for correlated metals that are close to a Fermi liquid phase, is the mass renormalization factor $Z$, which gives the ratio of the effective mass $m^{*}$ of the electrons to the band mass $m_{b}$ calculated from DFT, and is calculated from the slope of the DMFT electronic self-energy near the Fermi level as

$$
Z=\frac{m_{b}}{m^{*}}=\left.\left(1-\frac{\partial \operatorname{Re} \Sigma(\omega)}{\partial \omega}\right)^{-1}\right|_{\omega=0} .
$$

Earlier DMFT calculations and experiments agree that the parent compounds have mass renormalization factors of $Z_{\mathrm{SrVO} 3} \sim$ 0.5 and $Z_{\mathrm{SrNbO3}} \sim 0.7$ [17,43-45]. (This value is very well established for $\mathrm{SrVO}_{3}$, but it is harder to verify experimentally in $\mathrm{SrNbO}_{3}$ due to possible $\mathrm{Sr}$ deficiency of the samples.) In Fig. 2(a) we present the spectral function and density

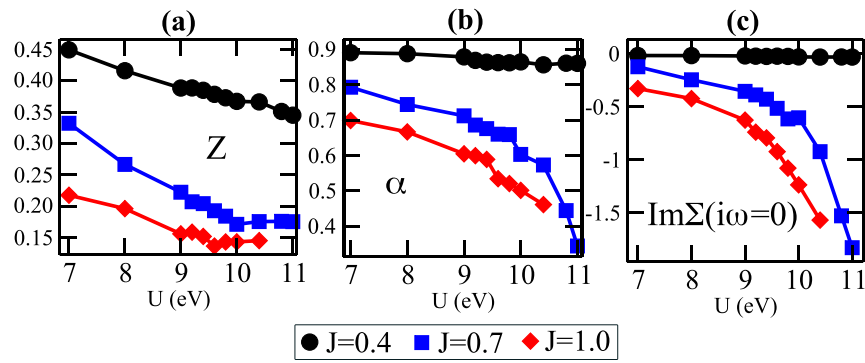

FIG. 3. Dependence of (a) the mass renormalization $Z$, (b) exponent of the real part of self-energy $\alpha$, and (c) zero-energy intercept of the imaginary part of the self-energy for $\mathrm{V} t_{2 g}$ orbitals in $r-\mathrm{Sr}_{2} \mathrm{VNbO}_{6}$ as a function of $U$ for different Hund's coupling $J$ values from DFT+DMFT, calculated in the crystal structure as determined from $\mathrm{DFT}+U$. For $J=1 \mathrm{eV}$, the system becomes insulating above $U=$ $10.4 \mathrm{eV}$.

of states of $\mathrm{Sr}_{2} \mathrm{VNbO}_{6}$. As expected, the $\mathrm{V}-t_{2 g}$ bands near $E_{\mathrm{F}}$ are narrower compared to those in DFT, and hence the double perovskite (with the crystal structure from DFT $+U$ ) is a highly correlated metal with $Z=0.16$ at room temperature according to DFT+DMFT. It also behaves as a non-Fermi liquid: The exponent $\alpha$ of the self-energy, which is $\alpha=1$ in ideal Fermi liquids, is $\alpha \sim 0.61$, which is a signature of Hund's metallic behavior $[21,46]$.

In order to elucidate the origin of strong correlations in double perovskite $\mathrm{Sr}_{2} \mathrm{VNbO}_{6}$, we repeat our DFT+eDMFT calculations using the same value of Hubbard $U$, but varying the value of Hund's coupling $J$. (Our results so far used $J=0.7 \mathrm{eV}$, a typical value for $\mathrm{V}$ in our implementation.) The results presented in Fig. 2(b) show that both $Z$ and $\alpha$ strongly depend on $J$. There is a more than twofold increase in the value of $Z$ with decreasing $J$. There is also a large increase in $\alpha$ with reduced $J$ and its gets larger than $\sim 0.90$ for small values of $J$. This behavior of $Z$ at room temperature is comparable to that observed in compounds such as $\mathrm{SrRuO}_{3}$ and $\mathrm{CaRuO}_{3}$, where the orbital degeneracy and the on-site Hund's coupling are important in determining the correlation strength [47]. Thus, the double perovskite $\mathrm{Sr}_{2} \mathrm{VNbO}_{6}$ can be classified as a Hund's metal, but only if the DFT $+U$ determination of crystal structural parameters used so far are accurate. In the following section we show that this is not the case, and $r-\mathrm{Sr}_{2} \mathrm{VNbO}_{6}$ is a Mott insulator when the crystal structure parameters are calculated taking electronic correlations into account as well.

To further elucidate the nature of the electronic correlations in metallic $r-\mathrm{Sr}_{2} \mathrm{VNbO}_{6}$, we present the evolution of mass renormalization $Z$, the self-energy exponent $\alpha$, and the intercept of the imaginary self-energy $\operatorname{Im}[\Sigma(i \omega=0)]$ for the $\mathrm{V}-t_{2 g}$ orbitals in Fig. 3 with the Hubbard $U$ for three different values of $J$. While we classify this system as a Hund's metal, it is very close to the boundary of the Mott insulating phase, and increasing the value of the Hubbard $U$ by $2 \mathrm{eV}$ while keeping $J=0.7 \mathrm{eV}$ drives the system insulating. The critical $U$ value is mildly reduced by increasing $J$ to $J=1.0 \mathrm{eV}$. Reducing the Hund's coupling to $J=0.4 \mathrm{eV}$, on the other hand, has a more dramatic effect: It not only increases $Z$ by more than a factor of 2 , but it also turns the system into a good Fermi liquid 

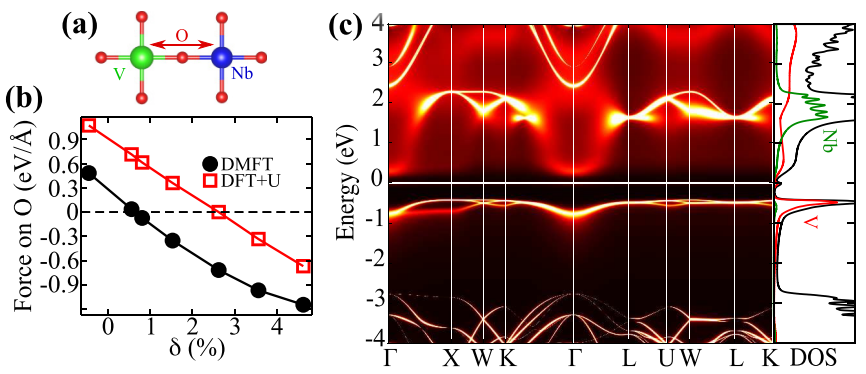

FIG. 4. (a) Only internal parameter in the crystal structure of $r-\mathrm{Sr}_{2} \mathrm{VNbO}_{6}$, the bond-length disproportionation. (b) Force on the $\mathrm{O}$ ions as a function of bond-length disproportionation $\delta$ calculated using DFT+DMFT, as well as DFT $+U$. The inset sketches how the only internal parameter $\delta$ changes the crystal structure. (c) Spectral function of $r-\mathrm{Sr}_{2} \mathrm{VNbO}_{6}$ from DFT+DMFT with $\delta$ also optimized using DFT+DMFT.

with zero intercept of the imaginary part of the self-energy and exponent $\alpha$.

\section{B. Crystal structure of $r-\mathrm{Sr}_{2} \mathrm{VNbO}_{6}$ from DMFT}

The common approach in DFT+DMFT studies is to use the crystal structure obtained either from experiments or from DFT $+U$ calculations, as we did so far. It has also become possible recently to obtain crystal structural parameters from DFT+DMFT using a stationary implementation $[30,35,48]$. In order to check if the DFT $U$ structural parameters are reliable in the room temperature paramagnetic phase, we optimized the ionic positions in rocksalt ordered $\mathrm{Sr}_{2} \mathrm{VNbO}_{6}$ using $\mathrm{DFT}+\mathrm{DMFT}$ as well. There is only one internal parameter to optimize, which is the $\mathrm{V}-\mathrm{O} / \mathrm{Nb}-\mathrm{O}$ bond-length disproportionation, shown in Fig. 4(a). We quantify this disproportionation as $\delta=\left(d_{\mathrm{Nb}-\mathrm{O}}-d_{\mathrm{V}-\mathrm{O}}\right) / d_{\mathrm{V}-\mathrm{O}}$. In Fig. $4(\mathrm{~b})$ we plot the force on the $\mathrm{O}$ atoms as a function of $\delta$ both from DFT+DMFT and $\mathrm{DFT}+U$ for comparison. DFT + DMFT predicts a much smaller $\delta$ than DFT $+U$, which is in-line with the very similar ionic radii of $\mathrm{V}^{3+}$ and $\mathrm{Nb}^{5+}$ [49]. This $2 \%$ reduction in $\delta$ has a strong effect on the electronic structure. The DFT+DMFT spectral function of $r-\mathrm{Sr}_{2} \mathrm{VNbO}_{6}$ with $\delta=0.6 \%$, shown in Fig. 4(c), indicates that this material is a Mott insulator. In other words, the effect of the electronic correlations on the crystal structure is strong enough to induce a transition from a Hund's metallic phase to a Mott insulating one in $r$ - $\mathrm{Sr}_{2} \mathrm{VNbO}_{6}$. The probabilities of $\mathrm{V}$ atomic impurity states (Fig. 5) show that this transition is accompanied by an increase in $\left\langle\left|S_{z}\right|\right\rangle$ for vanadium, which also emphasizes the importance of Hund's coupling.

In passing, we note that $r-\mathrm{Sr}_{2} \mathrm{VNbO}_{6}$ has vanadium $S=1$ moments on a face-centered-cubic lattice. This is similar to other double perovskites $\mathrm{La}_{2} \mathrm{LiReO}_{6}$ and $\mathrm{Ba}_{2} \mathrm{YReO}_{6}$ where implications of frustrated magnetism were discussed $[6,50]$. However, our DFT+DMFT calculations show no tendency for magnetic ordering at room temperature (the self-energy is identical for different orbitals and spins even when it is allowed to be unequal), which is expected due to the large distance and the lack of short superexchange pathways between the magnetic atoms in $r-\mathrm{Sr}_{2} \mathrm{VNbO}_{6}$. We also performed $\mathrm{DFT}+U$ calculations to determine any tendency of
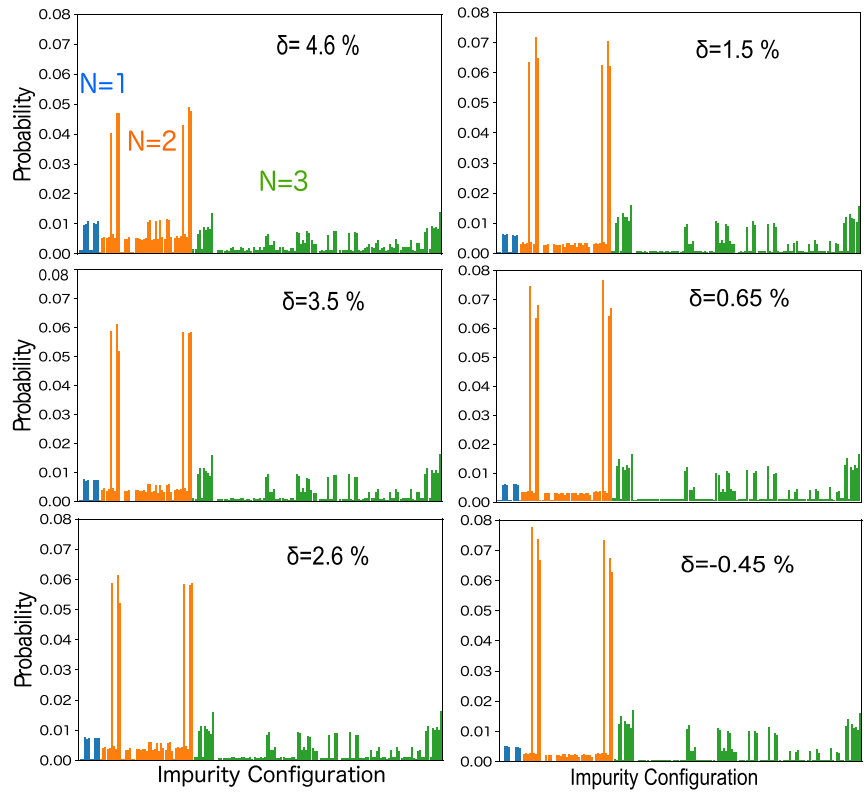

FIG. 5. Histogram of V impurity's $3 d$ states for different values of bond-length disproportionation in rocksalt ordered $\mathrm{Sr}_{2} \mathrm{VNbO}_{6}$. The $J$ parameter is $0.7 \mathrm{eV}$ in all plots. Different colors represent different total occupations of the V $3 d$ shell. The six high-probability $N=2$ configurations are the high-spin $\left(\left\langle\left|S_{z}\right|\right\rangle=1\right)$ states.

orbital ordering in $r-\mathrm{Sr}_{2} \mathrm{VNbO}_{6}$. Even for values as large as $U=4 \mathrm{eV}$, we could not stabilize an orbitally ordered configuration in the ferromagnetic phase. This is contrary to, for example, rhenium double perovskites, where orbital order can be stabilized for $U$ as small as $U=2.6 \mathrm{eV}$, even when crystal symmetry breaking is not taken into account [51]. When the spin-lattice coupling is omitted, DFT $+U$ predicts the lowest-energy magnetic structure to be antiferromagnetic with wave vector $k=(1,1 / 2,0)$ for a wide range of $U$ values. Unlike the ferromagnetic one, this magnetic order leads to an intricate orbital ordering pattern. We leave the detailed study of the orbital ordering in this $d^{2}-d^{0}$ double perovskite for future work.

\section{Layered ordered structure}

While the rocksalt is the most common ordering type for $B$-site ordered double perovskites, another possibility is layered ordering [7] [Fig. 1(b)]. In bulk double perovskites, layered ordering is often stabilized by Jahn-Teller effect, which is strong in cuprates such as $\mathrm{La}_{2} \mathrm{CuSnO}_{6}$ and $\mathrm{La}_{2} \mathrm{CuZrO}_{6}$ [52-54]. While $\mathrm{V}^{3+}$ has two electrons in its $t_{2 g}$ orbitals, and hence it can in principle lower its energy through a JahnTeller-type distortion, this tendency is expected to be much weaker than that in $\mathrm{Cu}^{2+}$ due to the difference in orbital characters. As a result, there is probably no driving force for layered ordering in bulk $\mathrm{Sr}_{2} \mathrm{VNbO}_{6}$. However, even for combinations of cations that form disordered solid solutions in bulk, layer-by-layer synthesis methods such molecular beam epitaxy make it possible to grow (001) heterostructures with periods as short as two unit cells, which can be considered as layered double perovskites (see, for example, Ref. [55].) The layered structure allows very different electronic properties 


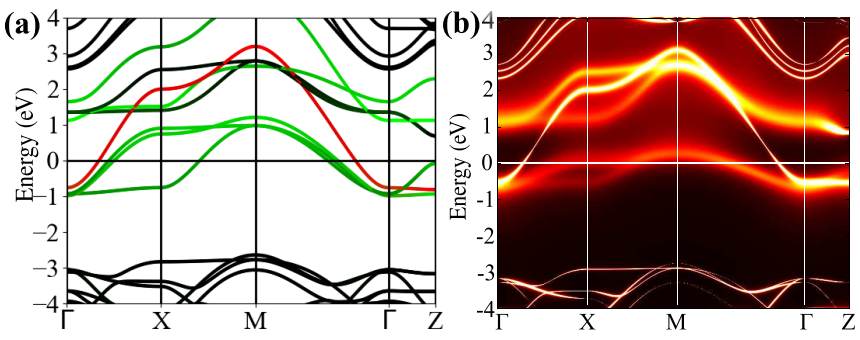

FIG. 6. (a) DFT and (b) DFT+DMFT electronic structure of $l$ $\mathrm{Sr}_{2} \mathrm{VNbO}_{6}$. In the DFT band structure, green and red represent the total $\mathrm{V}$ and $\mathrm{Nb} d_{x y}$ character of the bands.

since it hosts infinite two-dimensional planes of either perovskite parent, which was the reason that the cuprate double perovskites were studied in a high- $T_{C}$ superconductivity context $[53,54]$. We now turn our attention to the layered ordered compound, which we refer to as $l-\mathrm{Sr}_{2} \mathrm{VNbO}_{6}$.

In Fig. 6(a) we show the DFT band structure of $l$ $\mathrm{Sr}_{2} \mathrm{VNbO}_{6}$. Unlike $r-\mathrm{Sr}_{2} \mathrm{VNbO}_{6}$, where only $\mathrm{V}$ bands cross the Fermi level, $l-\mathrm{Sr}_{2} \mathrm{VNbO}_{6}$ has some $\mathrm{Nb}$ states at Fermi level in addition to the $\mathrm{V}$ states. This is due to the large bandwidth of the $\mathrm{Nb} d_{x y}$ band (where the $z$ axis is defined to be in the layering direction), which is highly dispersive in the $\mathrm{NbO}_{6}$ planes because of the unbroken $\mathrm{NbO}_{6}$ octahedral network in these planes. Thus, the $\mathrm{V}$ to $\mathrm{Nb}$ charge transfer is not complete in $l-\mathrm{Sr}_{2} \mathrm{VNbO}_{6}$. The differences in the DFT band structures of $l-\mathrm{Sr}_{2} \mathrm{VNbO}_{6}$ and $r-\mathrm{Sr}_{2} \mathrm{VNbO}_{6}$ get more emphasized when electronic correlations are taken into account via DFT+DMFT. In Fig. 6(b) we show the spectral function of $l-\mathrm{Sr}_{2} \mathrm{VNbO}_{6}$ obtained from DFT+DMFT using internal ionic positions also predicted by DFT+DMFT. (The electronic correlations have a smaller effect on the crystal structure of $l-\mathrm{Sr}_{2} \mathrm{VNbO}_{6}$ than they do on that of $r-\mathrm{Sr}_{2} \mathrm{VNbO}_{6}$.) As a result of increased bandwidth and incompleteness of the charge transfer, $l-\mathrm{Sr}_{2} \mathrm{VNbO}_{6}$ does not become a Mott insulator when correlations are taken into account, as seen from the DMFT spectral function shown in Fig. 6(b). This is a crucial difference between the two cation orderings: Even though $r-\mathrm{Sr}_{2} \mathrm{VNbO}_{6}$ and $l-\mathrm{Sr}_{2} \mathrm{VNbO}_{6}$ consist of the same building blocks, the two different cation orderings result in different charge states and electronic structures according to both DFT and DFT+DMFT calculations. The width of the $d_{x y}$ band is renormalized by a small amount in $l-\mathrm{Sr}_{2} \mathrm{VNbO}_{6}$ and its renormalization factor $Z_{\mathrm{Nb}-x y}=0.86$ is close to that of bulk $\mathrm{SrNbO}_{3}$. The $\mathrm{V}-t_{2 g}$ bands, on the other hand, exhibit stronger correlation effects with $Z_{V} \sim 0.30$ for all three orbitals. The value of $Z$ depends on the Hund's coupling $J$ as expected, albeit weakly compared to $r-\mathrm{Sr}_{2} \mathrm{VNbO}_{6}$ : For the same range of $J$ values, $Z$ changes by less than a factor of 2 and for $J=$ $0.7 \mathrm{eV}$ the exponent of the imaginary part of the vanadium self-energy is $\alpha_{V} \sim 0.84$, indicating mild Hund's metallic behavior [Fig. 7(a)].

Despite stronger correlations of the $\mathrm{V}-t_{2 g}$ electrons than those of the $\mathrm{Nb}, l-\mathrm{Sr}_{2} \mathrm{VNbO}_{6}$ is not a Mott insulator, unlike the rocksalt ordered $r-\mathrm{Sr}_{2} \mathrm{VNbO}_{6}$. This is due to the larger bandwidth of the transition metal bands crossing the Fermi level, which is in turn due to the connected layers of $\mathrm{VO}_{6}$ and $\mathrm{NbO}_{6}$ layers in the layered compound. In other words,
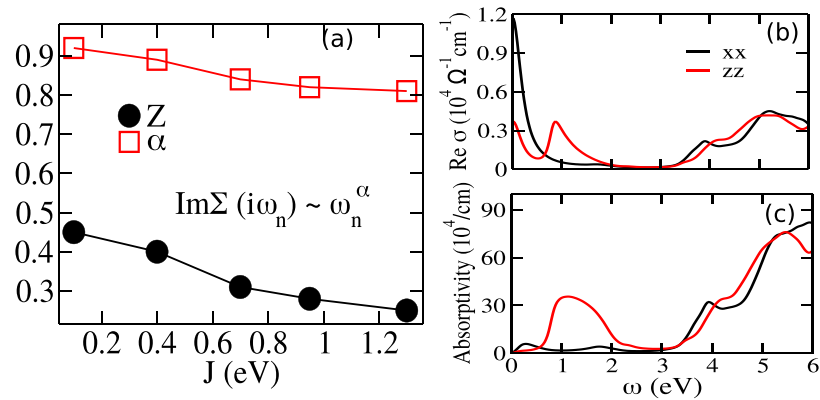

FIG. 7. (a) Quasiparticle weight $Z$ and the exponent of the imaginary part of the self-energy on the imaginary axis $\alpha$ of $l-\mathrm{Sr}_{2} \mathrm{VNbO}_{6}$ at room temperature for varying degrees of on-site Hund's coupling $J$. (b) Optical conductivity and (c) absorptivity spectra of $l-\mathrm{Sr}_{2} \mathrm{VNbO}_{6}$ computed at the DFT level. Unlike the parent perovskites, there is very strong absorption between $\sim 1$ and $2 \mathrm{eV}$, precluding the possibility of using $l-\mathrm{Sr}_{2} \mathrm{VNbO}_{6}$ as a transparent conductor.

different cation ordering gives rise to very different physical properties. One interesting question that arises at this point is what the effect of cation disorder, i.e., random arrangement of $\mathrm{V}$ and $\mathrm{Nb}$ cations, is. First-principles calculations that can simulate some degree of cation disorder require large supercells that are currently beyond the reach of DFT+DMFT due to their computational cost. Nevertheless, given that two different cations orders lead to metallic and insulating phases in $\mathrm{Sr}_{2} \mathrm{VNbO}_{6}$, we posit that a metal-insulator transition should exist as a function of degree of cation ordering in $\mathrm{Sr}_{2} \mathrm{VNbO}_{6}$.

\section{CORRELATED METALLIC TRANSPARENT CONDUCTORS}

There is high demand for compounds that are transparent to visible light and are good electrical conductors at the same time. Correlated metals can be promising as transparent conductors because of their large carrier concentration compared to doped semiconductors [56]. Both $\mathrm{SrVO}_{3}$ and $\mathrm{SrNbO}_{3}$ have been considered as correlated transparent conductors with different transparency windows, where $\mathrm{SrVO}_{3}$ is transparent on the lower-energy visible spectrum and $\mathrm{SrNbO}_{3}$ is transparent for blue and ultraviolet light $[17,43,56,57]$. A natural question to ask at this point is whether the layered $l-\mathrm{Sr}_{2} \mathrm{VNbO}_{6}$ double perovskite can bring together the positive aspects of both parent compounds and serve as a transparent metal that is transparent throughout the visible spectrum. Unfortunately, the optical conductivity [Figs. 7(b) and 7(c)] of $l-\mathrm{Sr}_{2} \mathrm{VNbO}_{6}$ shows that this is not the case, because a wide absorption peak at $\sim 1.1 \mathrm{eV}$ emerges in the layered compound, hampering its use as a transparent conductor. However, a disordered or partially ordered $\mathrm{Sr}_{2} \mathrm{VNbO}_{6}$ is likely to not have this absorption peak because of the lack of translational symmetry breaking and might be more promising as a transparent conductor.

\section{SUMMARY AND CONCLUSIONS}

We performed first-principles DFT+DMFT calculations on double perovskites of $\mathrm{Sr}_{2} \mathrm{VNbO}_{6}$ with different (rocksalt and layered) cation orderings. Even though $\mathrm{V}$ and $\mathrm{Nb}$ are both group 5 transition metals, there is almost complete 
charge transfer between them when they coexist in the double perovskite. The two different double perovskites obtained by different orderings of $\mathrm{V}$ and $\mathrm{Nb}$ cations have starkly different electronic properties: The layered compound is a correlated metal with a mild or moderate degree of Hund's metallicity, whereas the rocksalt ordered compound is a Mott insulator with $S=1$ moments on a frustrated fcc lattice.

Our results underline two points that can be generalized to double perovskites beyond $\mathrm{Sr}_{2} \mathrm{VNbO}_{6}$ : (i) Intercationic charge transfer, which is quite common in double perovskites, can exist even between cations from the same column of the Periodic Table and can give rise to very different chemistries than the parent perovskites and (ii) the strength of electronic correlations in double perovskites, as measured by $Z$ in our approach, can be tuned by the cation order. This might be a fruitful way to obtain new correlated metallic perovskite compounds. Finally, our results on $\mathrm{Sr}_{2} \mathrm{VNbO}_{6}$ adds Hund's metallicity to the list of phenomena that emerges in double perovskites even when it does not exist in parent compounds.

\section{ACKNOWLEDGMENTS}

This work was supported by NSF DMREF Grant No. DMR-1629260. We acknowledge the Minnesota Supercomputing Institute at the University of Minnesota for providing resources that contributed to the research results reported within this paper.
[1] Y. Tokura and N. Nagaosa, Science 288, 462 (2000).

[2] S.-W. Cheong, Nat. Mater. 6, 927 (2007).

[3] C. N. R. Rao, Annu. Rev. Phys. Chem. 40, 291 (1989).

[4] D. Khomskii and G. Sawatzky, Solid State Commun. 102, 87 (1997).

[5] D. I. Khomskii, Transition Metal Compounds (Cambridge University Press, Cambridge, 2014).

[6] S. Vasala and M. Karppinen, Prog. Solid State Chem. 43, 1 (2015).

[7] G. King and P. M. Woodward, J. Mater. Chem. 20, 5785 (2010).

[8] S. F. Matar, M. A. Subramanian, A. Villesuzanne, V. Eyert, and M. H. Whangbo, J. Magn. Magn. Mater. 308, 116 (2007).

[9] K. I. Kobayashi, T. Kimura, H. Sawada, K. Terakura, and Y. Tokura, Nature (London) 395, 677 (1998).

[10] H. Kato, T. Okuda, Y. Okimoto, Y. Tomioka, Y. Takenoya, A. Ohkubo, M. Kawasaki, and Y. Tokura, Appl. Phys. Lett. 81, 328 (2002).

[11] M. Sakai, A. Masuno, D. Kan, M. Hashisaka, K. Takata, M. Azuma, M. Takano, and Y. Shimakawa, Appl. Phys. Lett. 90, 072903 (2007).

[12] H. Das, M. De Raychaudhury, and T. Saha-Dasgupta, Appl. Phys. Lett. 92, 201912 (2008).

[13] O. Mustonen, S. Vasala, E. Sadrollahi, K. P. Schmidt, C. Baines, H. C. Walker, I. Terasaki, F. J. Litterst, E. Baggio-Saitovitch, and M. Karppinen, Nat. Commun. 9, 1085 (2018).

[14] P. Davies, H. Wu, A. Borisevich, I. Molodetsky, and L. Farber, Annu. Rev. Mater. Res. 38, 369 (2008).

[15] C. Meneghini, S. Ray, F. Liscio, F. Bardelli, S. Mobilio, and D. D. Sarma, Phys. Rev. Lett. 103, 046403 (2009).

[16] D. D. Sarma, Curr. Opin. Solid State Mater. Sci. 5, 261 (2001).

[17] A. Paul and T. Birol, Phys. Rev. Mater. 3, 085001 (2019).

[18] K. Haule, C.-H. Yee, and K. Kim, Phys. Rev. B 81, 195107 (2010).

[19] A. Paul and T. Birol, Annu. Rev. Mater. Res. 49, 31 (2019).

[20] G. Kotliar and D. Vollhardt, Phys. Today 57(3), 53 (2004).

[21] X. Deng, K. M. Stadler, K. Haule, A. Weichselbaum, J. von Delft, and G. Kotliar, Nat. Commun. 10, 2721 (2019).

[22] K. Haule and G. Kotliar, New J. Phys. 11, 025021 (2009).

[23] G. Kresse and J. Furthmuller, Comput. Mater. Sci. 6, 15 (1996).

[24] G. Kresse and J. Furthmüller, Phys. Rev. B 54, 11169 (1996).
[25] J. P. Perdew, A. Ruzsinszky, G. I. Csonka, O. A. Vydrov, G. E. Scuseria, L. A. Constantin, X. Zhou, and K. Burke, Phys. Rev. Lett. 100, 136406 (2008).

[26] M. Rey, P. Dehaudt, J. Joubert, B. Lambert-Andron, M. Cyrot, and F. Cyrot-Lackmann, J. Solid State Chem. 86, 101 (1990).

[27] D. Ridgley and R. Ward, J. Am. Chem. Soc. 77, 6132 (1955).

[28] S. L. Dudarev, G. A. Botton, S. Y. Savrasov, C. J. Humphreys, and A. P. Sutton, Phys. Rev. B 57, 1505 (1998).

[29] P. Blaha, K. Schwarz, G. K. H. Madsen, D. Kvasnicka, J. Luitz, R. Laskowski, F. Tran, and L. D. Marks, WIEN2k: An Augmented Plane Wave Program for Calculating Crystal Properties (Vienna University of Technology, Vienna, 2018).

[30] K. Haule and G. L. Pascut, Phys. Rev. B 94, 195146 (2016).

[31] E. Gull, A. J. Millis, A. I. Lichtenstein, A. N. Rubtsov, M. Troyer, and P. Werner, Rev. Mod. Phys. 83, 349 (2011).

[32] K. Haule, Phys. Rev. B 75, 155113 (2007).

[33] K. Haule, Phys. Rev. Lett. 115, 196403 (2015).

[34] K. Haule, T. Birol, and G. Kotliar, Phys. Rev. B 90, 075136 (2014).

[35] K. Haule and T. Birol, Phys. Rev. Lett. 115, 256402 (2015).

[36] K. Li and D. Xue, J. Phys. Chem. A 110, 11332 (2006).

[37] H. Chen and A. Millis, J. Phys.: Condens. Matter 29, 243001 (2017).

[38] K. Kuepper, M. Raekers, C. Taubitz, H. Hesse, M. Neumann, A. T. Young, C. Piamonteze, F. Bondino, and K. C. Prince, J. Appl. Phys. 104, 036103 (2008).

[39] P. Karen, A. R. Moodenbaugh, J. Goldberger, P. N. Santhosh, and P. M. Woodward, J. Solid State Chem. 179, 2120 (2006).

[40] H. Chen and A. Millis, Sci. Rep. 7, 6142 (2017).

[41] H. Chen, D. P. Kumah, A. S. Disa, F. J. Walker, C. H. Ahn, and S. Ismail-Beigi, Phys. Rev. Lett. 110, 186402 (2013).

[42] T. Miruszewski, B. Kamecki, M. Łapiński, and J. Karczewski, Mater. Chem. Phys. 212, 446 (2018).

[43] J. Roth, A. Paul, N. Goldner, A. Pogrebnyakov, K. Agueda, T. Birol, N. Alem, and R. Engel-Herbert, ACS Appl. Mater. Interfaces 12, 30520 (2020).

[44] T. Yoshida, K. Tanaka, H. Yagi, A. Ino, H. Eisaki, A. Fujimori, and Z.-X. Shen, Phys. Rev. Lett. 95, 146404 (2005).

[45] C. Taranto, M. Kaltak, N. Parragh, G. Sangiovanni, G. Kresse, A. Toschi, and K. Held, Phys. Rev. B 88, 165119 (2013).

[46] Z. P. Yin, K. Haule, and G. Kotliar, Phys. Rev. B 86, 195141 (2012). 
[47] H. T. Dang, J. Mravlje, A. Georges, and A. J. Millis, Phys. Rev. B 91, 195149 (2015).

[48] K. Haule, J. Phys. Soc. Jpn. 87, 041005 (2018).

[49] R. D. Shannon, Acta Crystallogr. A 32, 751 (1976).

[50] T. Aharen, J. E. Greedan, C. A. Bridges, A. A. Aczel, J. Rodriguez, G. MacDougall, G. M. Luke, V. K. Michaelis, S. Kroeker, C. R. Wiebe, H. Zhou, and L. M. D. Cranswick, Phys. Rev. B 81, 064436 (2010).

[51] A. T. Lee and C. A. Marianetti, Phys. Rev. B 97, 045102 (2018).

[52] K. Samanta and T. Saha-Dasgupta, Phys. Rev. B 95, 235102 (2017).

[53] M. Azuma, S. Kaimori, and M. Takano, Chem. Mater. 10, 3124 (1998).
[54] M. T. Anderson, K. R. Poeppelmeier, S. A. Gramsch, and J. K. Burdett, J. Solid State Chem. 102, 164 (1993).

[55] T. S. Santos, B. J. Kirby, S. Kumar, S. J. May, J. A. Borchers, B. B. Maranville, J. Zarestky, S. G. E. te Velthuis, J. van den Brink, and A. Bhattacharya, Phys. Rev. Lett. 107, 167202 (2011).

[56] L. Zhang, Y. Zhou, L. Guo, W. Zhao, A. Barnes, H.-T. Zhang, C. Eaton, Y. Zheng, M. Brahlek, H. F. Haneef, N. J. Podraza, M. H. W. Chan, V. Gopalan, K. M. Rabe, and R. Engel-Herbert, Nat. Mater. 15, 204 (2016).

[57] Y. Park, J. Roth, D. Oka, Y. Hirose, T. Hasegawa, A. Paul, A. Pogrebnyakov, V. Gopalan, T. Birol, and R. Engel-Herbert, Commun. Phys. 3, 102 (2020). 